\title{
Association of the 553 or GSTM1 polymorphism with the risk of nasopharyngeal carcinoma: A meta-analysis
}

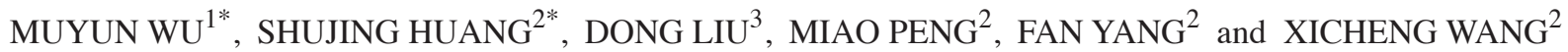 \\ ${ }^{1}$ Department of Oncology, The Fifth People's Hospital of Wuhu, Wuhu, Anhui 241000; ${ }^{2}$ Department of Oncology, \\ First Affiliated Hospital of Guangdong Pharmaceutical University, Guangzhou, Guangdong 510080; \\ ${ }^{3}$ Department of Internal Medicine, Guangdong Pharmaceutical University, Guangzhou, Guangdong 510006, P.R. China
}

Received May 11, 2015; Accepted November 12, 2015

DOI: $10.3892 / \operatorname{mco} .2015 .700$

\begin{abstract}
S-transferase M1 (GSTM1) are the most popular suppressor genes. Several previous studies demonstrated positive associations of these gene polymorphisms with numerous cancer types, including hepatocellular cancer, while the association between p53/GSTM1 polymorphisms and the nasopharyngeal carcinoma (NPC) risk was inconsistent and underpowered. However, no studies investigating the combinational effect of these two genes on NPC risk were performed. To confirm the effects of p53 and GSTM1 polymorphisms on the risk of NPC, a meta-analysis of all the available previous studies associating p53 and GSTM1 with the risk of NPC was performed. A comprehensive search of PubMed, Web of Science and SD database until November 2014 was performed to identify the relevant studies. The data were extracted by two independent authors and pooled odds ratio (OR) with 95\% confidence interval (CI) was calculated. Meta-regression and subgroup analyses were performed to identify the source of heterogeneity. Finally, five studies with 1,419 cases and 1,707 controls were included for the $\mathrm{p} 53$ polymorphism and three studies with 837 cases and 1,299 controls were included for the GSTM1 polymorphism. Regarding p53, a significantly increased NPC risk was observed in the overall population (C vs. G, OR, 1.245; 95\% CI, 1.045-1.483; $\mathrm{P}=0.014$; additive models: CC vs. GG, OR, 1.579; 95\% CI, 1.100-2.265; P=0.013 and $\mathrm{CG}$ vs. GG, OR, 1.230; 95\% CI, 1.039-1.456; $\mathrm{P}=0.016$; dominant model, OR, 1.321; 95\% CI, 1.127-1.549; $\mathrm{P}=0.001$; recessive model, OR, 1.429; 95\% CI, 1.017-2.009; $\mathrm{P}=0.040$ ). Concerning GSTM1, a significantly increased NPC risk was
\end{abstract}

Correspondence to: Mr. Xicheng Wang, Department of Oncology, First Affiliated Hospital of Guangdong Pharmaceutical University, Guangzhou, Guangdong 510080, P.R. China

E-mail: 13902400598@126.com

*Contributed equally

Key words: p53, GSTM1, polymorphism, meta-analysis, nasopharyngeal carcinoma observed in the overall population (null versus non-null, OR, 1.282; 95\% CI, 1.075-1.530; $\mathrm{P}=0.006$ ). In the subgroup analyses stratified by the source of controls, a significant association of p53 with NPC risk was also demonstrated, while no association with GSTM1 was observed. Therefore, the p53 G72C polymorphism may have a susceptible role in the carcinogenesis of NPC, while genetic deletion of GSTM1 may contribute to increased susceptibility to NPC. Further large and well-designed studies are required to confirm this association.

\section{Introduction}

Nasopharyngeal carcinoma (NPC) is one of the most common types of cancer worldwide, with a high frequency of nodal and distant metastasis at diagnosis. The incidence is high in South China and East Asia; however, it is rare in the Western world (1-4). According to epidemiological studies, Epstein-Barr virus (EBV) infection and the environment are two risk factors for the pathogenesis of NPC $(5,6)$. Certain previous studies show that the morbidity of NPC remains high among Chinese individuals who have migrated to North America, which indicates that host genetic factors, including the genetic alterations of tumor suppressor genes and oncogenes, have an important role in the pathogenesis of NPC $(7,8)$. The present study observed that p53 and glutathione S-transferase M1 (GSTMI) were the most commonly studied suppressor genes.

The p53 gene, located on chromosome 17p13, is a tumor suppressor gene, which is critical in maintaining genomic stability and regulating the cell cycle. The mutant p53 gene may contribute to the development of malignant tumors (9-11). There is a single base change of $\mathrm{G}$ to $\mathrm{C}$ at codon 72 in exon 4 of the p53 gene, known as the p53 G72C polymorphism (rs1042522). This change causes an alteration of the amino acid residue from arginine (Arg) to proline (Pro). These two polymorphisms are likely candidate genetic markers of certain types of cancer $(12,13)$.

Another common tumor suppressor gene is a member of the GSTs, which constitute a superfamily of ubiquitous, multifunctional enzymes and have a critical role in cellular detoxification. GSTM1, located on chromosome 1p13.3, is known to be highly polymorphic. GSTM1 is a homologous recombination, involving left and right $4.2 \mathrm{~kb}$ repeats and resulting in a $16 \mathrm{~kb}$ deletion, containing the entire GSTM1 gene. 
This genetic variation may change the susceptibility of an individual to carcinogens and toxins, an identical affect on the toxicity and efficacy of certain drugs (14-16).

Although numerous previous studies have been performed to investigate p53 and GSTM1 genetic variation with respect to NPC, the results are somewhat controversial and underpowered. Therefore, a meta-analysis of all the available case-control studies associating p53 or GSTM1 gene polymorphisms with the risk of developing NPC is required to derive a more precise estimation of the association between the p53/GSTM1 polymorphism and NPC risk. Due to the high incidence rate, the present study focused on East Asian populations.

\section{Materials and methods}

Search strategy. A comprehensive search strategy was performed using the electronic databases, including PubMed (http://www.ncbi.nlm.nih.gov/pubmed/), Web of Science (http://wokinfo.com/) and SD database (http://www.sciencedirect.com/), with a search strategy based on combinations of the keywords 'nasopharyngeal, rhinopharyngeal, nasopharynx, rhinopharynx or nasal part of pharynx;' and 'cancer, tumor, tumour, neoplasm, carcinoma or adenocarcinoma;' and 'polymorphism, variation, genetic, mutation, variant or SNP;' and 'P53, TP53, GSTM1 or Glutathione S-tranferase M1'. The last search was updated on November 1, 2014. Although no language restrictions were applied initially, for the full-text review and final analysis, the resources only permitted the review of studies published in English. Reference lists of the identified studies were also examined and literature retrieval was performed in duplication by two independent reviewers. When multiple publications reported identical or overlapping data, the most recent or largest population was selected. When a study reported the results on different subpopulations, the studies were treated as separate studies in the meta-analysis.

Selection criteria. Abstracts of all the citations and retrieved studies were reviewed. The following criteria were used to include published studies :i) Evaluating the association between p53/GSTM1 gene polymorphism and NPC; ii) case-control design; iii) the studies must offer the size of the samples, distribution of alleles, genotypes or other information, which can aid in inferring the results to estimate the odds ratio (OR) and their $95 \%$ confidence intervals (CIs); iv) participants were from East Asia, including China, Japan and Korea; and v) studies published in English. The age of the individuals was not included in the exclusion criteria. Previous studies were excluded if one of the following existed: i) A review, case report, editorial or comment; ii) the genotype frequency was not reported; iii) laboratory molecular or animal studies; iv) there was insufficient information for data extraction.

Data extraction. The data from the eligible studies that were selected in strict accordance with the inclusion criteria were independently extracted by two investigators. The controversial issues were resolved following discussion. The following data were extracted from each study: The first author's name, publication year, country of origin, genotyping method, source of control, total number of cases and controls, genotype frequen-
Table I. Scale for quality assessment.

Criteria

\section{Representativeness of cases}

Selected from population or cancer registry 3

Selected from hospital

Selected from pathology archives (no description)

Not described

Credibility of controls

Population-based

Blood donors or volunteers

Hospital-based (cancer-free patients)

Not described

Specimens of cases determining genotypes

White blood cells or normal tissues

Tumor tissues or exfoliated cells of tissue

Hardy-Weinberg equilibrium in controls

Hardy-Weinberg equilibrium

Hardy-Weinberg disequilibrium

Total sample size

$$
\begin{aligned}
& \geq 1,000 \\
& \geq 400 \text { and }<1,000 \\
& \geq 200 \text { and }<400 \\
& <200
\end{aligned}
$$

1

cies of cases and controls, and the P-value for Hardy-Weinberg equilibrium (HWE) test in the controls.

Quality score assessment. The two investigators independently assessed the quality of the eligible studies, according to a set of predefined criteria (Table I), which was originally proposed by Thankkinstian et al (17). The revised criteria cover the representativeness of cases, the credibility of controls, specimens of cases determining genotypes, HWE in the controls and the total sample size (Table I). The disagreements between two investigators were resolved by consensus. The total scores ranged between 0 (lowest) and 15 (highest). Previous studies with scores $\geq 10$ were considered as high-quality studies, while those with scores $<10$ were considered low-quality studies.

Statistical analysis. Summary ORs and corresponding 95\% CIs were used to estimate the strength of the associations between each polymorphism and NPC risk in different comparison models. As there were only two genotypes (null and non-null) for the GSTM1 gene, the pooled ORs were performed only between the two genotypes. By contrast, three genotypes (CC, $\mathrm{CG}$ and $\mathrm{GG}$ ) were observed for the p53 gene, and the pooled ORs were performed for the allele (C vs. $\mathrm{G})$, additive (CC vs. GG and CG vs. GG), dominant (CC + CG vs. GG) and recessive ( $\mathrm{CC}$ vs. $\mathrm{CG}+\mathrm{GG})$ models.

The statistical heterogeneity among studies was assessed by the Q test and $\mathrm{I}^{2}$ statistics $(18,19)$. A random-effects model (DerSimonian and Laird method) was used to estimate the summary ORs when the result of the $\mathrm{Q}$ test was $\mathrm{P}_{\mathrm{Q}}<0.1$ or $\mathrm{I}^{2} \geq 50 \%$, which indicated the presence of heterogeneity. The 




Figure 1. Flow diagram of the study identification.

fixed-effects model (Mantel-Haenszel method) was used when the result of the $\mathrm{Q}$ test was $\mathrm{P}_{\mathrm{Q}} \geq 0.1$ and $\mathrm{I}^{2}<50 \%$, which indicated the absence of heterogeneity (20,21). Logistic meta-regression and subgroup analyses were performed to assess the sources of heterogeneity among the studies. Subgroup analysis was performed by source of the controls.

The leave-one-out sensitivity analysis was performed to identify whether omitting each study had a major influence on the pooled estimates. Publication bias was assessed using the Begg's funnel plot and Egger's test $(22,23)$. The Duval and Tweedie non-parametric 'trim and fill' method was used to adjust for publication bias when it existed (24). The distribution of the genotypes in the control population was tested for HWE using a goodness-of-fit $\chi^{2}$ test. The Stata software, version 11.0 (Stata Corp., College Station, TX, USA) was used for all statistical analyses. All P-values were two-sided and all data were entered into the statistical software programs by two authors independently, with identical results ensuring the reliability and the accuracy of the results.

\section{Results}

Study characteristics. Subsequent to performing a careful search, 322 potentially relevant publications were identified. Based on the inclusion criteria, 8 studies ( 5 for p53 and 3 for GSTM1) (25-32) were eligible for this meta-analysis. A flow diagram describing the selection process for the eligible studies is shown in Fig. 1. The predominant characteristics of the studies about p53 are presented in Table II, and those about GSTM1 are presented in Table III. A total of 5 studies, including 1,419 cases and 1,707 controls for p53 gene polymorphisms, and 3 studies, including 837 cases and 1,299 controls for GSTM1 gene polymorphism. The sample size in these studies varied considerably, ranging between 109 and 1,234 individuals. Of all the eligible studies, 8 were performed in China, each of the p53 and GSTM1 studies had 1 study performed in the Taiwanese population $(26,30)$. For p53, 3 studies were population-based (PB) and 2 were hospital-based (HB), and for GSTM1, 2 studies were PB and 1 was HB. Several genotyping methods were used, including polymerase chain reaction (PCR), PCR-restriction fragment length polymorphism and PCR-confronting two-pair primers. As not all the studies provided HWE information, the expected distribution was calculated using the observed data, and the genotype distribution among controls in the 5 case-control studies did not deviate from the HWE, as the P-values for estimation of the HWE were all $>0.05$, as shown in Table II. With respect to GSTM1, as only two genotypes (null and non-null) were included in the 3 studies, the HWE could not be evaluated. In the case of the assessment of the quality of the included studies, as shown in Table II, 2 were identified as 'low quality' $(25,26)$, and 3 were identified as 'high quality' (27-29). As shown in Table III, all the studies were identified as 'high quality' (30-32).

Meta-analysis results. For the p53 G72C polymorphism, a significantly increased NPC risk was observed in all the genetic models in the overall population (C vs. G, OR, 1.245; 95\% CI, 1.045-1.483; $\mathrm{P}=0.014$; additive model: $\mathrm{CC}$ vs. $\mathrm{GG}$, OR, 1.579; 95\% CI, 1.100-2.265; P=0.013; CG vs. GG, OR, 1.230; 95\% CI, 1.039-1.456; $\mathrm{P}=0.016$; dominant model, OR, 1.321; 95\% CI, 1.127-1.549; $\mathrm{P}=0.001$; recessive model, OR, 1.429; 95\% CI, 1.017-2.009; P=0.040; Table IV and Fig. 2). According to the subgroup analysis stratified by source of controls, significantly increased NPC risk was observed among PB studies for the additive model (CG vs. GG, OR, 1.266; 95\% CI, 1.061-1.511; P=0.009) and dominant model (CC + CG vs. GG, OR, 1.327; 95\% CI, 1.123-1.569; $\mathrm{P}=0.001)$, while the increased risk was observed among $\mathrm{HB}$ studies for the allele model (C vs. G, OR, 1.505; 95\% CI, 1.065-2.127; $\mathrm{P}=0.020$ ), additive model (CC vs. GG, $\mathrm{OR}, 2.188 ; 95 \% \mathrm{CI}, 1.136-4.212 ; \mathrm{P}=0.019)$ and recessive model (CC vs. CG + GG, OR, 2.188; 95\% CI, 1.250-3.830; $\mathrm{P}=0.006$; Table IV and Fig. 2).

For the GSTM1 gene polymorphism, a significantly increased NPC risk was observed in the overall population (null versus non-null, OR, 1.282; 95\% CI, 1.075-1.530; $\mathrm{P}=0.006$; Table IV and Fig. $2 \mathrm{~F}$ ), the subgroup analysis used the source of controls. As there was only $1 \mathrm{HB}$ study case-control design, stratified analysis was only conducted in studies with a PB case-control design, and there was no association between the GSTM1 polymorphism and NPC risk among PB studies (Table IV and Fig. 2F).

Heterogeneity analysis. The heterogeneity by $\mathrm{Q}$ statistical tests was significant in the p53 allele model $\left(\mathrm{C}\right.$ vs. $\mathrm{G}, \mathrm{P}_{\mathrm{Q}}=0.056$ and $\mathrm{I}^{2}=56.7 \%$ ), additive model ( $\mathrm{CC}$ vs. $\mathrm{GG}, \mathrm{P}_{\mathrm{Q}}=0.050$ and $\mathrm{I}^{2}=57.7 \%$ ) and recessive model (CC vs. $\mathrm{CG}+\mathrm{GG}: \mathrm{P}_{\mathrm{Q}}=0.021$ and $\left.\mathrm{I}^{2}=65.2 \%\right)$; therefore, the random-effects model was selected to calculate the pooled results in these data and the fixed-effects model was used to calculate the others. When analyzing the subgroups of these models by source of controls, the heterogeneity was also significant in these models among PB studies (Table IV).

The heterogeneity was not significant for GSTM1, and the fixed-effects model was selected to calculate the pooled results in the meta-analysis. No significant heterogeneity was observed in the subgroups by source of controls (Table IV). 


A



C

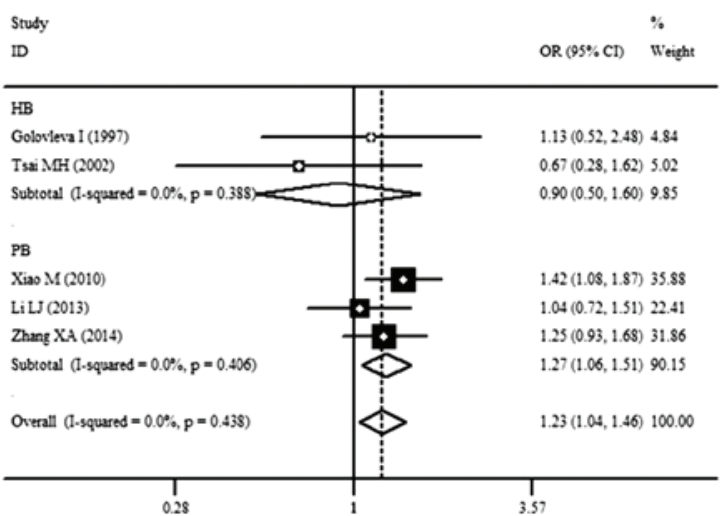

E

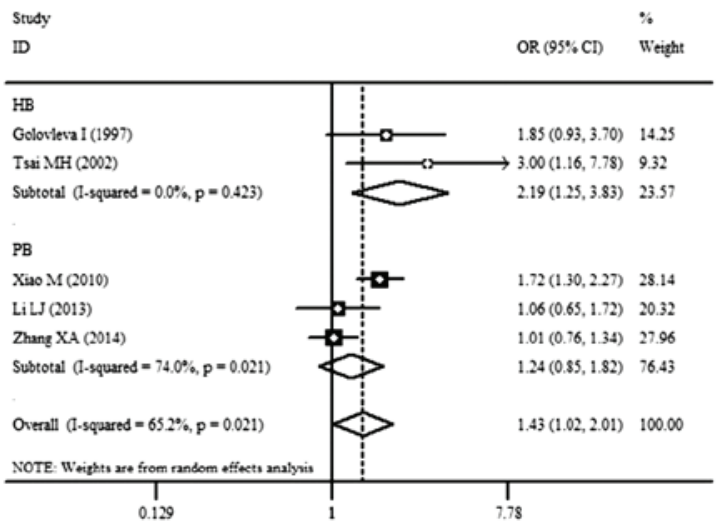

B



D

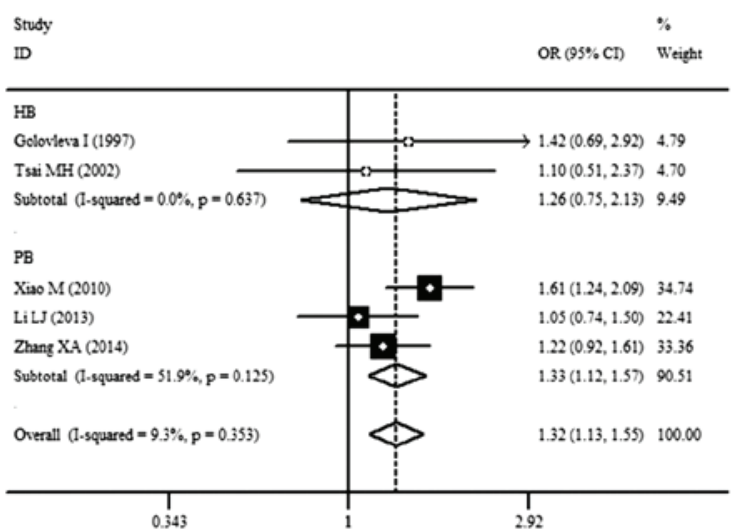

F



Figure 2. Forest plots for the association of p53 and GSTM1 gene polymorphisms with the risk of NPC. (A) p53 allele model, C vs. G; (B) p53 additive model, CC vs. GG; (C) p53 additive model, CG vs. GG; (D) p53 dominant model, CC + CG vs. GG; (E) p53 recessive model, CC vs. CG + GG; (F) GSTM1, null versus non-null. OR, odds ratio; $\mathrm{PB}$, population-based; $\mathrm{HB}$, hospital-based; CI, confidence intervals.

Sensitivity analysis. A single study was deleted from the overall pooled analysis each time to assess the effect of the removed data set to the overall ORs. The pooled ORs and 95\% CIs were not significantly changed when any section of the study was omitted, which indicated that any single study had little impact on the overall ORs.

Publication bias. Begg's funnel plot and Egger's test were used to assess the publication bias of literatures in all the comparison models. Symmetrical funnel plots were obtained in the single-nucleotide polymorphism (SNP) tested in the whole models. Egger's test further validated the absence of publication bias in this meta-analysis ( $\mathrm{P}>0.05$; Fig. 3 ).

\section{Discussion}

NPC is a common head and neck neoplasm in East Asia with a complicated etiology. Numerous susceptible genes have been demonstrated to be involved in the risk of NPC, including TGF 31 , DNA repair genes and interleukin-12p40 $(8,28,33,34)$. Following assessment of previous studies, it was determined that the most common gene polymorphisms previously studied were p53 and GSTM1.p53 and GSTM1 are regulators for genotoxic stress, including DNA damage, DNA repair and cell cycle regulation. Mutations in these genes may influence their normal function and thereby effect on the development of malignant tumors $(9,14)$. The association between the 
Table IV. Meta-analysis of the p53 and GSTM1 gene polymorphisms on nasopharyngeal carcinoma risk.

\begin{tabular}{|c|c|c|c|c|c|c|c|c|c|}
\hline \multirow[b]{2}{*}{ Polymorphism } & \multirow[b]{2}{*}{ Population } & \multirow[b]{2}{*}{ Studies, $\mathrm{n}$} & \multicolumn{3}{|c|}{ Test of association } & \multirow[b]{2}{*}{ Model } & \multicolumn{3}{|c|}{ Test of heterogeneity } \\
\hline & & & OR & $95 \% \mathrm{CI}$ & P-value & & $\chi^{2}$ & $\mathrm{P}_{\mathrm{Q}^{- \text {value }}}$ & $\mathrm{I}^{2}, \%$ \\
\hline \multicolumn{10}{|l|}{ p53 } \\
\hline \multirow[t]{3}{*}{ C vs. G } & Overall & 5 & 1.245 & $1.045-1.483$ & 0.014 & $\mathrm{R}$ & 9.23 & 0.056 & 56.70 \\
\hline & PB & 3 & 1.187 & $0.956-1.474$ & 0.121 & $\mathrm{R}$ & 7.89 & 0.019 & 74.70 \\
\hline & HB & 2 & 1.505 & $1.065-2.127$ & 0.020 & $\mathrm{~F}$ & 0.01 & 0.912 & 0.00 \\
\hline \multirow[t]{3}{*}{ CC vs. GG } & Overall & 5 & 1.579 & $1.100-2.265$ & 0.013 & $\mathrm{R}$ & 9.47 & 0.050 & 57.70 \\
\hline & $\mathrm{PB}$ & 3 & 1.433 & $0.904-2.272$ & 0.126 & $\mathrm{R}$ & 8.24 & 0.016 & 75.70 \\
\hline & $\mathrm{HB}$ & 2 & 2.188 & $1.136-4.212$ & 0.019 & $\mathrm{~F}$ & 0.11 & 0.743 & 0.00 \\
\hline \multirow[t]{3}{*}{ CG vs. GG } & Overall & 5 & 1.230 & $1.039-1.456$ & 0.016 & $\mathrm{~F}$ & 3.77 & 0.438 & 0.00 \\
\hline & PB & 3 & 1.266 & $1.061-1.511$ & 0.009 & $\mathrm{~F}$ & 1.80 & 0.406 & 0.00 \\
\hline & $\mathrm{HB}$ & 2 & 0.898 & $0.502-1.604$ & 0.715 & $\mathrm{~F}$ & 0.75 & 0.388 & 0.00 \\
\hline \multirow[t]{3}{*}{$\mathrm{CC}+\mathrm{CG}$ vs. $\mathrm{GG}$} & Overall & 5 & 1.321 & $1.127-1.549$ & 0.001 & $\mathrm{~F}$ & 4.41 & 0.353 & 9.30 \\
\hline & $\mathrm{PB}$ & 3 & 1.327 & $1.123-1.569$ & 0.001 & $\mathrm{~F}$ & 4.16 & 0.125 & 51.90 \\
\hline & $\mathrm{HB}$ & 2 & 1.263 & $0.748-2.131$ & 0.382 & $\mathrm{~F}$ & 0.22 & 0.637 & 0.00 \\
\hline \multirow[t]{3}{*}{$\mathrm{CC}$ vs. $\mathrm{CG}+\mathrm{GG}$} & Overall & 5 & 1.429 & $1.017-2.009$ & 0.040 & $\mathrm{R}$ & 11.50 & 0.021 & 65.20 \\
\hline & PB & 3 & 1.245 & $0.852-1.819$ & 0.258 & $\mathrm{R}$ & 7.70 & 0.021 & 74.00 \\
\hline & $\mathrm{HB}$ & 2 & 2.188 & $1.250-3.830$ & 0.006 & $\mathrm{~F}$ & 0.64 & 0.423 & 0.00 \\
\hline \multicolumn{10}{|l|}{ GSTM1 } \\
\hline \multirow[t]{3}{*}{ null versus non-null } & Overall & 3 & 1.282 & $1.075-1.530$ & 0.006 & $\mathrm{~F}$ & 1.58 & 0.454 & 0.00 \\
\hline & PB & 2 & 1.202 & $0.981-1.474$ & 0.076 & $\mathrm{~F}$ & 0.01 & 0.904 & 0.00 \\
\hline & $\mathrm{HB}$ & 1 & 1.563 & $1.094-2.232$ & 0.014 & $\mathrm{~F}$ & 0.00 & - & - \\
\hline
\end{tabular}

OR, odds ratio; $\mathrm{CI}$, confidence intervals; $\mathrm{P}_{\mathrm{Q}}$-value, $\mathrm{P}$-value for the $\mathrm{Q}$ test; $\mathrm{R}$, random-effects model; $\mathrm{F}$, fixed-effects model; $\mathrm{PB}$, population-based; HB, hospital-based.

A

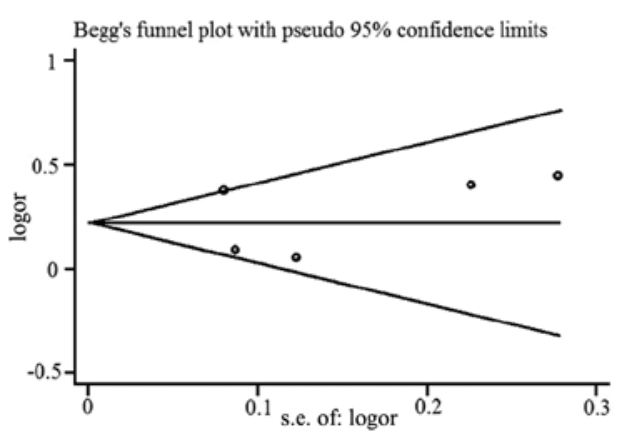

C

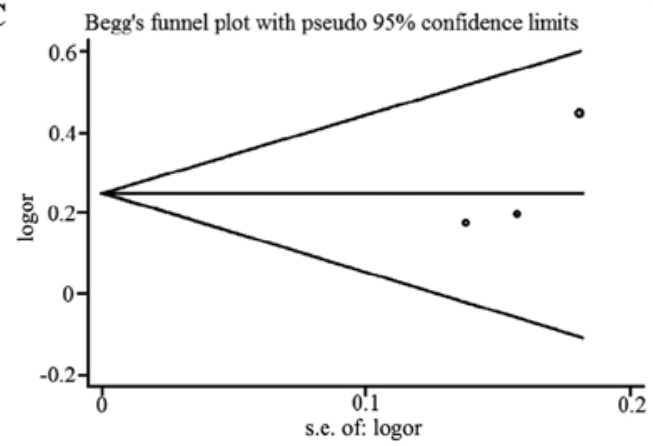

B

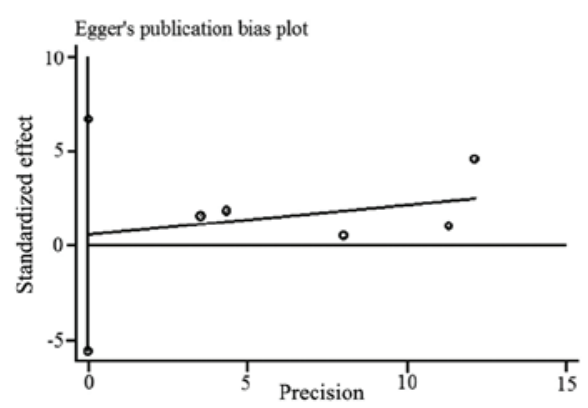

D

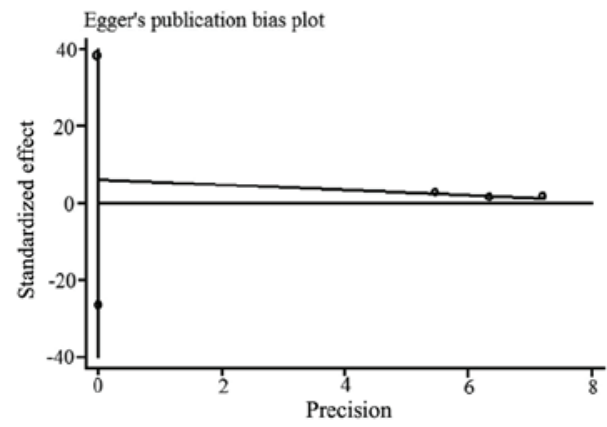

Figure 3. Begg's funnel plot and Egger's test analyses to detect publication bias. Each point represents a separate previous study for the indicated association. (A) Begg's funnel plot for allele C vs. G for p53 gene polymorphism in overall analysis; (B) Egger's test for allele C vs. G for p53 gene polymorphism in overall analysis; (C) Begg's funnel plot for GSTM1 gene polymorphism in overall analysis; (D) Egger's test for GSTM1 gene polymorphism in overall analysis. s.e., standard error. 
p53/GSTM1 gene polymorphism and NPC risk was inconsistent and underpowered in these previous studies. However, no studies regarding the combinatorial effect of the two genes on NPC risk were identified. Additionally, there are certain previous studies investigating the association between the p53/GSTM1 gene polymorphisms and neoplasms, including oral, cervical and hepatocellular cancer. Certain results are positive, while others are negative (35-40). Therefore, the association between the p53/GSTM1 gene polymorphisms and NPC risk remains to be elucidated.

Xiao et al (27) revealed that an increased NPC risk was associated with the p53 CC genotype compared with the GG genotype, which was the same result as Tsai et al (26). However, Zhang et al (29) failed to identify any association between the p53 gene polymorphism and NPC risk. In addition, Jiang et al (32) identified that a genetic deletion of GSTM1 contributed to increased susceptibility to NPC. However, the investigation by Guo et al (31) into the GSTM1 gene polymorphism and NPC risk was a negative result. Therefore, it is worthy to perform a meta-analysis to assess the association between p53/GSTM1 gene polymorphism and NPC risk. With regards to the high incidence in East Asia, the present study focused on the p53/GSTM1 gene polymorphisms and NPC risk studies in the Chinese, Japanese and Korean populations. A total of 5 studies, including 1,419 cases and 1,707 controls for the p53 gene polymorphism, and 3 studies, including 837 cases and 1,299 controls for the GSTM1 gene polymorphism were included. Significantly increased NPC risks were observed for the p53 G72C and GSTM1 gene polymorphisms in all the genetic models in the overall population. In the subgroup analysis based on the source of controls, a significantly increased NPC risk of the p53 gene polymorphism was observed among the PB studies for the additive and dominant models, while the increased risk was observed among the HB studies for the allele, additive and recessive models. However, in the analysis of subgroup stratified by source of controls, no association between the GSTM1 gene polymorphism and NPC risk was observed.

As p53 and GSTM1 are the most studied suppressor genes, certain studies have analyzed these genes. Zhou et al (41) performed a meta-analysis on the association of the p53 polymorphism with NPC risk, and identified that an Arg allele markedly decreased the NPC risk, however, it failed to prove that the Pro allele increased the NPC risk (41). In 2014, Ren et al (42) demonstrated a negative result following analysis of the association between p53 and head and neck carcinoma risks, including the NPC risk. In 2014, Cai et al (43) indicated that the change of the p53 gene from Arg to Pro increased the susceptibility to NPC in all the gene models, with the exception of the additive model (CG vs. GG). With respect to the GSTM1 polymorphism, 4 studies published between 2009 and 2013 performed meta-analyses on the association of the GSTM1 polymorphism with the NPC risk and revealed an identical result, which was that GSTM1 null genotypes increased the NPC risk (44-47). The present study included new information regarding the association of the p53 and GSTM1 gene polymorphisms with the NPC risk up until November 2014. The present study focused on East Asian populations considering the high incidence in
East Asia, and demonstrated positive results in all the gene models, which had the largest sample size and provided more accurate research for prevention and individualized treatment.

Sensitivity analysis revealed that omitting any single study had no significant impact on the combined ORs. The Begg's funnel plot reflected no clear asymmetry and the Egger's test further indicated no considerable publication bias in the meta-analysis. Notably, certain limitations remained in the meta-analysis. Firstly, the meta-analysis was based on published studies only, which led to publication bias. Secondly, the meta-analysis focused on the studies in the East Asian population published in English and the eligible studies were all from the Chinese population. Further studies are required to investigate the subgroups by ethnicity. Thirdly, the overall sample sizes of p53 and GSTM1 were not large enough. Future studies are required to enhance the reliability and accuracy of these conclusions. Fourthly, meta-analysis is a case-control study. In the present meta-analysis, certain studies were PB, while others were HB. The controls were not uniformly defined and although the majority of the control groups were selected from healthy populations, certain individuals may have harbored a benign disease. Fifthly, the genotyping methods used were not identical in all the studies assessed. Additionally, the quality scores of these studies were not all high quality. Sixthly, other clinical factors were not shown, including age, gender, stage and type, which may lead to bias. The analysis failed to consider gene-gene and gene-environment interactions due to the lack of sufficient data. The infection of EBV, consumption of a high-salt diet, tobacco smoking and alcohol consumption are also risk factors for NPC. Finally, for the GSTMI gene polymorphism, only two genotypes exist (null and non-null), so the lack of data prevented HWE assessment.

In conclusion, the present meta-analysis suggested that the p53 G72C polymorphism may have a susceptible role in the carcinogenesis of NPC in the Chinese population, while genetic deletion of GSTM1 may contribute to increased susceptibility to NPC in the Chinese population. However, further large and well-designed studies considering gene-gene and gene-environment interactions, and using standardized genotyping methods and well-matched controls are required to confirm this association and lead to an improved comprehensive understanding of the association between the p53/GSTM1 gene polymorphism and NPC risk.

\section{References}

1. Lee AW, Ng WT, Chan YH, Sze H, Chan C and Lam TH: The battle against nasopharyngeal cancer. Radiother Oncol 104: 272-278, 2012.

2. Bei JX, Li Y, Jia WH, Feng BJ, Zhou G, Chen LZ, Feng QS, Low HQ, Zhang H, He F, et al: A genome-wide association study of nasopharyngeal carcinoma identifies three new susceptibility loci. Nat Genet 42: 599-603, 2010.

3. Chang CM, Yu KJ, Mbulaiteye SM, Hildesheim A and Bhatia K: The extent of genetic diversity of Epstein-Barr virus and its geographic and disease patterns: A need for reappraisal. Virus Res 143: 209-221, 2009.

4. Cho WC: Nasopharyngeal carcinoma: Molecular biomarker discovery and progress. Mol Cancer 6: 1, 2007.

5. Horikawa T, Yoshizaki T, Kondo S, Furukawa M, Kaizaki Y and Pagano JS: Epstein-Barr virus latent membrane protein 1 induces snail and epithelial-mesenchymal transition in metastatic nasopharyngeal carcinoma. Br J Cancer 104: 1160-1167, 2011 
6. Hildesheim A and Wang CP: Genetic predisposition factors and nasopharyngeal carcinoma risk: A review of epidemiological association studies, 2000-2011: Rosetta Stone for NPC: Genetics, viral infection and other environmental factors. Semin Cancer Biol 22: 107-116, 2012.

7. Su CK and Wang CC: Prognostic value of Chinese race in nasopharyngeal cancer. Int J Radiat Oncol Biol Phys 54: 752-758, 2002.

8. Qin HD, Shugart YY, Bei JX, Pan QH, Chen L, Feng QS, Chen LZ, Huang W, Liu JJ, Jorgensen TJ, et al: Comprehensive pathway-based association study of DNA repair gene variants and the risk of nasopharyngeal carcinoma. Cancer Res 71: 3000-3008, 2011.

9. Levine AJ and Oren M: The first 30 years of p53: Growing ever more complex. Nat Rev Cancer 9: 749-758, 2009.

10. Goodsell DS: The molecular perspective: p53 tumor suppressor. Oncologist 4: 138-139, 1999.

11. Hickman ES and Helin K: The p53 tumor suppressor protein. Biotechnol Genet Eng Rev 17: 179-211, 2000.

12. Jiang P, Liu J, Zeng X, Li W and Tang J: Association of TP53 codon 72 polymorphism with cervical cancer risk in Chinese women. Cancer Genet Cytogenet 197: 174-178, 2010.

13. Xiong X, Wang M, Wang L, Liu J, Zhao X, Tian Z and Wang J: Risk of MDM2 SNP309 alone or in combination with the p53 codon 72 polymorphism in acute myeloid leukemia. Leuk Res 33: 1454-1458, 2009.

14. Hayes JD and Pulford DJ: The glutathione S-transferase supergene family: Regulation of GST and the contribution of the isoenzymes to cancer chemoprotection and drug resistance. Crit Rev Biochem Mol Biol 30: 445-600, 1995.

15. Parl FF: Glutathione S-reansferase genotypes and cancer risk. Cancer Lett 221: 123-129, 2005.

16. Scheneider J, Bernges U, Philipp M and Woitowitz HJ: GSTM1, GSTT1 and GSTP1 polymorphism and lung cancer risk in relation to tobacco smoking. Cancer Lett 208: 65-74, 2004.

17. Thakkinstian A, McEvoy M, Minelli C, Gibson P, Hancox B, Duffy D, Thompson J, Hall I, Kaufman J, Leung TF, et al: Systematic review and meta-analysis of the association between \{beta\}2-adrenoceptor polymorphisms and asthma: A HuGE review. Am J Epidemiol 162: 201-211, 2005.

18. Higgins JP and Thompson SG: Quantifying heterogeneity in a meta-analysis. Stat Med 21: 1539-1558, 2002.

19. Higgins JP, Thompson SG, Deeks JJ and Altman DG: Measuring inconsistency in meta-analyses. BMJ 327: 557-560, 2003.

20. DerSimonian R and Laird N: Meta-analysis in clinical trials. Control Clin Trials 7: 177-188, 1986.

21. Mantel N and Haenszel W: Statistical aspects of the analysis of data from retrospective studies of disease. J Natl Cancer Inst 22 719-748, 1959.

22. Egger M, Davey Smith G, Schneider M and Minder C: Bias in meta-analysis detected by a simple, graphical test. BMJ 315 : 629-634, 1997.

23. Stuck AE, Rubenstein LZ and Wieland D: Bias in meta-analysis detected by a simple, graphical test. Asymmetry detected in funnel plot was probably due to true heterogeneity. BMJ 316 : 470-471, 1998.

24. Duval S and Tweedie R: Trim and fill: A simple funnel-plot-based method of testing and adjusting for publication bias in meta-analysis. Biometrics 56: 455-463, 2000

25. Golovleva I, Birgander R, Själander A, Lundgren E and Beckman L: Interferon-alpha and p53 alleles involved in nasopharyngeal carcinoma. Carcinogenesis 18: 645-647, 1997.

26. Tsai MH, Lin CD, Hsieh YY, Chang FC, Tsai FJ, Chen WC and Tsai CH: Prognostic significance of the proline form of p53 codon 72 polymorphism in nasopharyngeal carcinoma. Laryngoscope 112: 116-119, 2002.

27. Xiao M, Zhang L, Zhu X, Huang J, Jiang H, Hu S and Liu Y: Genetic polymorphisms of MDM2 and TP53 genes are associated with risk of nasopharyngeal carcinoma in a Chinese population. BMC Cancer 10: 147, 2010

28. Li L, Wu J, Sima X, Bai P, Deng W, Deng X, Zhang L and Gao L: Interactions of miR-34b/c and TP-53 polymorphisms on the risk of nasopharyngeal carcinoma. Tumor Biol 34: 1919-1923, 2013.
29. Zhang XA, Chen X, Zhai Y, Cui Y, Cao P, Zhang H, Wu Z, Li P, Yu L, Xia X, et al: Combined effects of genetic variants of the PTEN, AKT1, MDM2 and p53 genes on the risk of nasopharyngeal carcinoma. PLoS One 9: e92135, 2014.

30. Cheng YJ, Chien YC, Hildesheim A, Hsu MM, Chen IH, Chuang J, Chang J, Ma YD, Luo CT, Hsu WL, et al: No association between genetic polymorphisms of CYP1A1, GSTM1, GSTT1, GSTP1, NAT2 and nasopharyngeal carcinoma in Taiwan. Cancer Epidemiol Biomarkers Prev 12: 179-180, 2003.

31. Guo XC, O'Brien SJ, Zeng Y, Nelson GW and Winkler CA GSTM1 and GSTT1 gene deletions and the risk for nasopharyngeal carcinoma in Han Chinese. Cancer Epidemiol Biomarkers Prev 17: 1760-1763, 2008

32. Jiang Y, Li N, Dong P, Zhang N, Sun Y, Han M, Wen J and Chen M: Polymorphisms in GSTM1, GSTT1 and GSTP1 and nasopharyngeal cancer in the east of China: A case-control study. Asian Pac J Cancer Prev 12: 3097-3100, 2011.

33. Hu S, Zhou G, Zhang L, Jiang H and Xiao M: The effects of functional polymorphisms in the TGF $\beta 1$ gene on nasopharyngeal carcinoma susceptibility. Otolaryngol Head Neck Surg 146: 579-584, 2012

34. Ben Chaaben A, Busson M, Douik H, Boukouaci W, Mamoghli T, Chaouch L, Harzallah L, Dorra S, Fortier C, Ghanem A, et al: Association of IL-12p40+1188A/C polymorphism with nasopharyngeal cancer risk and tumor extension. Tissue Antigens 78 : 148-151, 2011.

35. Chang KW, Lin SC, Mangold KA, Jean MS, Yuan TC, Lin SN and Chang CS: Alterations of adenomatous polyposis Coli (APC) gene in oral squamous cell carcinoma. Int J Oral Maxillofac Surg 29: 223-226, 2000

36. Rosenthal AN, Ryan A, Al-Jehani RM, Storey A, Harwood CA and Jacobs IJ: P53 codon 72 polymorphism and risk of cervical cancer in UK. Lancet 352: 871-872, 1998.

37. Wang NM, Tsai $\mathrm{CH}$, Yeh KT, Chen SJ and Chang JG: P53 codon 72Arg polymorphism is not a risk factor for carcinogenesis in the Chinese. Int J Mol Med 4: 249-252, 1995.

38. Zhuo W, Wang Y, Zhuo X, Zhu Y, Wang W, Zhu B, Li D and Chen Z: CYP1A1 and GSTM1 polymorphisms and oral cancer risk: Association studies via evidence-based meta-analyses. Cancer Invest 27: 86-95, 2009.

39. Gao LB, Pan XM, Li LJ, Liang WB, Bai P, Rao L, Su XW, Wang T, Zhou B, Wei YG, et al: Null genotypes of GSTM1 and GSTT1 contribute to risk of cervical neoplasia: An evidence-based meta-analysis. PLoS One 6: e20157, 2011.

40. White DL, Li D, Nurgalieva Z and El-Serag HB: Genetic variants of glutathione $S$-transferase as possible risk factors for hepatocellular carcinoma: A HuGE systematic review and meta-analysis. Am J Epidemiol 167: 377-389, 2008.

41. Zhou XL, Cai L, Xiang ZL, Zhou WL, Wang Y and Zhang XY: TP53 codon 72 polymorphism contributes to nasopharyngeal cancer susceptibility: A meta-analysis. Arch Med Res 40: 299-305, 2008.

42. Ren WH, Jiang DK, Pei Y, Wang SQ, Yang XM and Yu L: Meta-analysis of associations between the TP53 Arg72Pro polymorphism with risk of head and neck carcinomas based on case-control studies. Genet Mol Res 13: 103-114, 2014.

43. Cai K, Wang Y, Zhao X and Bao X: Association between the P53 codon 72 polymorphism and nasopharyngeal cancer risk. Tumor Biol 35: 1891-1897, 2014.

44. Zhou X, Cai L, Xiang Z, Li Q and Zhang X: GSTM1 and GSTT1 polymorphisms and nasopharyngeal cancer risk: An evidence-based meta-analysis. J Exp Clin Cancer Res 28: 46, 2009.

45. Sun ZF, Zhang J, Xu HM, Wang GL and Dong P: Association between GSTM1 polymorphism and nasopharyngeal cancer susceptibility: A meta-analysis. Asian Pac J Cancer Prev 13: 5817-5821, 2012

46. Wei Y,Zhou T, Lin H, Sun M, Wang D, Li H and Li B: Significant associations between GSTM1/GSTT1 polymorphisms and nasopharyngeal cancer risk. Tumor Biol 34: 887-894, 2013.

47. Murthy AK, Kumar V and Suresh KP: Meta-analysis of GSTM1 and GSTT1 polymorphisms and risk of nasopharyngeal cancer. Asian Pac J Cancer Prev 14: 1697-1701, 2013. 